\title{
VOCABULARIO GASTRONÓMICO EN EL HABLA INFANTIL COSTARRICENSE
}

\author{
Gastronomic vocabulary in Costa Rican children's language
}

\author{
Marielos Murillo Rojas ${ }^{1}$
}

\begin{abstract}
RESUMEN
Este estudio identifica el vocabulario gastronómico infantil mediante tres fuentes de información: el corpus lingüístico del lenguaje infantil del proyecto de investigación 745-B6-320, INIL-UCR, los resultados de entrevistas aplicadas a dependientes de pequeños comercios sobre las demandas alimenticias de los niños, a un grupo de niños preescolares y a sus respectivos docentes. Al triangular la información, se obtiene un inventario léxico conformado por vocabulario relativo a nombres de golosinas, frutas, comidas preparadas, bebidas, verduras, carnes, productos lácteos, cereales, nombres de marcas comerciales, condimentos e ingredientes necesarios para preparar alimentos. El inventario léxico obtenido es útil para diferentes disciplinas, entre ellas: la educación preescolar, la industria alimenticia, la nutrición, la lingüística aplicada, educativa y clínica.
\end{abstract}

Palabras clave: vocabulario infantil, nombres de alimentos, vocabulario gastronómico, adquisición de la lengua.

\section{SUMMARY}

This study identifies the children's gastronomic vocabulary through three sources of information: the linguistic corpus of children's language from research project 745-B6-320, INIL-UCR, the results of interviews applied to retailers about the food demands of children, the results of interviews applied to children and to their respective teachers. The lexical inventory obtained consists of names: candies, fruits, prepared foods, beverages, vegetables, meats, dairy products, cereals, trade names, condiments and food preparation ingredients. This lexical inventory is useful for different disciplines, including: preschool education, food industry, nutrition, applied linguistics, educational and clinical linguistics.

Key Words: children's vocabulary, food names, gastronomic vocabulary, language acquisition.

\section{Introducción}

Este estudio considera como vocabulario gastronómico en el habla infantil toda aquella unidad léxica que se relacione con nombres de alimentos, ya sea nombres de platos preparados, bebidas, verduras, frutas, golosinas, condimentos, productos necesarios para la cocción, productos lácteos, entre otros.

Tal como lo presentan Sánchez y Murillo (2006) el lenguaje da información sobre la experiencia que tiene el hablante acerca de la vida, objetos, animales, personas que lo rodean,

\footnotetext{
${ }^{1}$ Universidad de Costa Rica. Escuela de Formación Docente. Costa Rica. Correo electrónico: marielosmuro@ gmail.com

Recepción: 18-09-2017 Aceptación: 13-10-2017
} 
bienes, recursos, comidas, bebidas, vestimenta; en fin, informa sobre la memoria y conciencia de los hablantes. El niño conoce la lengua de su medio, la lengua que utiliza y oye a diario, la lengua con la que juega, con la que comparte los períodos de alimentación, con la que construye su propio mundo y su fantasía. Ante esta realidad, la institución educativa debe partir de los saberes ya adquiridos, a fin de activar aún más este proceso natural de enriquecimiento. Estas consideraciones justifican la pertinencia de este estudio y conceptualizan los momentos de alimentación como espacios de aprendizaje, de transmisión cultural, de disfrute, de enriquecimiento del vocabulario y también como respuesta a una necesidad biológica.

Al circunscribirse en la experiencia de vida infantil y en el proceso de adquisición de la lengua, se asume, tal como lo plantea Aguado (2000, p. 19), que este es "un trabajo compartido entre el niño y el adulto, pero es un trabajo de sol a sol". De esta manera, se resalta la importancia del entorno familiar y social como parte del input lingüístico necesario para el desarrollo del lenguaje.

El proceso de adquisición del lenguaje para Tomasello (2005) se da en virtud de una compleja interacción entre la herencia genética, la maduración biológica, el desarrollo cognitivo y la relación con el medio socioafectivo; dicho de otra manera, la capacidad para adquirir el lenguaje está dada por la presencia de un cerebro que madura articuladamente con las interacciones sociales en las que se utiliza el lenguaje.

Las interacciones sociales proporcionan las experiencias necesarias para que el niño amplíe su vocabulario; aceleran, por tanto, el tránsito entre las primeras palabras puramente referenciales hasta el uso de palabras que denotan y connotan. Es así como la adquisición del vocabulario está asociada con experiencias repetidas de acontecimientos que se producen cotidianamente, entre ellos, la alimentación (Cfr. Bruner, 1983).

De acuerdo con Ogden (2005), las actitudes de los padres frente a la comida y la elección de estas son fundamentales en el proceso de aprendizaje. Los alimentos que se compran para la casa y que se consumen en ella influyen en la conducta de alimentación de los niños y, por tanto, en el vocabulario gastronómico que conocen y utilizan.

La publicidad y la televisión también son factores básicos que condicionan el aprendizaje social de la alimentación en los niños. Unido a esto están los espacios en que se 
comparten períodos de alimentación en las instituciones educativas, en este caso en las preescolares.

En términos generales, la comida, además de constituirse en una necesidad biológica, es una práctica social, es una manifestación de la cultura, un rasgo que marca el estatus social de las personas, una fuente de placer y, en el caso de los niños, se utiliza también como una recompensa: "si se porta bien, le doy una galleta", "si se come toda la comida, le doy un regalito".

En este contexto, se pretende identificar el vocabulario gastronómico que usan los niños en su cotidianeidad, utilizando como fuentes de información el corpus del lenguaje infantil del proyecto de investigación 745-B6-320, Test morfosintáctico para niños costarricenses menores de 6 años, inscrito en el Instituto de Investigaciones Lingüísticas de la Universidad de Costa Rica, así como tres entrevistas: 1) entrevista aplicada a dependientes o pulperos, acerca de los productos alimenticios que compran los niños en tiendas de alimentos o pulperías, supermercados, abastecedores y minisúper; 2) entrevista realizada a un grupo niños sobre sus gustos alimenticios y 3) entrevista aplicada a docentes de Educación Preescolar respecto de los alimentos consumidos en las meriendas. Toda esta información permitirá identificar el vocabulario gastronómico que utilizan los niños en su cotidianeidad.

\section{Aspectos teóricos}

\subsubsection{El desarrollo del sentido del gusto en el niño}

El sabor es una amalgama compleja de información sensitiva proporcionada por el gusto, el olfato y la sensación táctil que tiene la comida cuando se mastica. En general, los científicos asocian la percepción humana del gusto a cuatro sabores: salado, dulce, ácido y amargo, pero se han sugerido otras categorías, entre ellas el umami, variedad gustativa que induce el glutamato, uno de los veinte aminoácidos que forman las proteínas de la carne, del pescado y las legumbres (Cfr. Smith y Margolskee, 2001).

De acuerdo con Moreno Villaresa y Galiano Segovia (2006), las papilas gustativas aparecen alrededor de la séptima u octava semana de gestación y son similares morfológicamente a las del adulto. Estos autores señalan que en estudios recientes realizados 
con niños en procesos de gestación (pretérminos, de 33 a 37 semanas), mediante un procedimiento de administración de pequeñas cantidades de productos por vía oral, se ha encontrado una preferencia por los sabores dulces. Siguiendo el curso del desarrollo, los recién nacidos muestran una fuerte aceptación por los sabores dulces, expresada por medio de muecas faciales de agrado ante la presencia de sustancias dulces y otras de rechazo o de incomodidad ante sabores agrios o amargos. Esta apetencia por los sabores dulces "se acompaña de efectos calmantes, experiencia que se ha aprovechado para usar como analgésico en recién nacidos la administración oral de una pequeña cantidad de sacarosa” (p.16).

La estimulación con sabores salados, tal como muestra la investigación citada, no produce ninguna respuesta facial específica en el recién nacido. No obstante, a partir del cuarto mes, el niño muestra también preferencias por este sabor.

El desarrollo del sentido del gusto más allá del período neonatal no ha sido suficientemente explorado, pero pareciera que persiste la preferencia por los sabores dulces y se da una progresiva aceptación por los agrios y salados. A partir de las reacciones no aprendidas a los sabores básicos, se establecen los patrones posteriores de aceptación de los alimentos.

\subsubsection{Las preferencias alimenticias de los niños}

Sullivan y Birch (1994), citado por Moreno Villaresa y Galiano Segovia (2006) y Rubio Navarro et al. (2013), señalan que los lactantes alimentados con leche materna experimentan los sabores de alimentos y especias que consume la madre y, por tanto, se adaptan más rápidamente a los nuevos alimentos que los que fueron alimentados con una fórmula infantil.

En este orden, Birch (1998) señala que, después de la apetencia por lo dulce, la segunda dimensión que afecta a las preferencias del lactante es la familiaridad. Los niños tienden a preferir los alimentos que les son familiares frente a los que no lo son. La transición de una dieta láctea a una diversificada requiere que el lactante aprenda a aceptar algunos de los nuevos alimentos que se le ofrecen. Etapa que podría estar afectada por la "neofobia", o 
sea, una respuesta negativa a la ingesta de nuevos alimentos, situación que implica una logística familiar para superar el rechazo de alimentos necesarios para el desarrollo y la salud del niño. La constancia familiar evitará que el niño consuma pocos alimentos y con un rango de diversificación reducido.

En este proceso de aprendizaje, es fundamental que el niño reciba los nuevos alimentos en espacios sociales positivos y sin excesivas restricciones a sus preferencias. Regularmente, los alimentos más apetecibles, con buen sabor y elevado contenido en grasa, azúcar y sal, se presentan en contextos sociales positivos, mientras que los alimentos menos llamativos, pero más saludables, se muestran en contextos sociales negativos, por ejemplo, cuando se les obliga a comer.

Con frecuencia las prácticas sociales que giran en torno a la alimentación harán que el niño desee no consumir los alimentos sugeridos por sus padres y, al contrario, se incline por alimentos de elevado contenido energético, sobre todo cuando tienen hambre. Unido a lo señalado, la toma de decisiones del niño respecto de la alimentación puede estar influida por la publicidad o los medios de comunicación masiva.

Por otra parte, la fácil disponibilidad de alimentos de alto contenido energético, su asociación con contextos sociales positivos, la predisposición infantil hacia esos alimentos y el hecho de que los más sabrosos son los que producen menos sensación de saciedad, condicionan las preferencias alimenticias de los niños en el presente y predefinen su futuro.

La certeza de que los niños consumen alimentos no recomendados por desfavorecer su proceso de desarrollo y salud justifica la presencia de una serie de investigaciones que se ocupan de la educación nutricional en la niñez y la adolescencia, del papel de la publicidad en la fijación de hábitos alimenticios, de las carencias nutricionales de los niños y de las estadísticas de hábitos de consumo, entre otros. Para efectos del presente trabajo, se retoman los principales hallazgos del estudio exploratorio Promoción y publicidad de alimentos y bebidas no saludables dirigida a niños en América Latina y el Caribe (2013), impulsado por UNICEF en colaboración con el Instituto de Nutrición de Centro América y Panamá (INCAP), estudio que interesa particularmente debido a que los datos para valorar la situación de la región incluyen tres países y uno de ellos es Costa Rica. 
En términos generales, los datos muestran que hasta octubre de 2014 el $31 \%$ de los países de la región tiene normas que regulan la promoción de alimentos y bebidas. La mayoría de sitios web y redes sociales revisadas poseían técnicas de promoción y publicidad dirigidas a niños: Facebook es el canal más utilizado. Las caricaturas, imágenes de niños menores de 16 años y claves visuales relacionadas con la música, deporte, videojuegos, cine o juegos clásicos fueron las técnicas dirigidas hacia los niños. En el interior de las escuelas evaluadas, la publicidad indirecta fue la más frecuente, la cual se da mediante artículos gratuitos o regalías y actividades patrocinadas por las empresas. Además, en todas las sodas (pequeños establecimientos de comidas y bebidas) al interior de las escuelas se encontraron disponibles alimentos y bebidas no saludables (p. ej. galletas dulces/saladas o pasteles, chocolates, etc.) y el $58 \%$ de las 16 escuelas observadas tenía kioscos y vendedores ambulantes afuera de sus instalaciones y, finalmente, el $25 \%$ algún tipo de promoción y publicidad externa.

En vista de las evidencias obtenidas se ha reconocido a la promoción y publicidad de alimentos y bebidas no saludables (es decir que contienen altas cantidades de grasas, azúcar o sodio) como uno de los principales contribuyentes y factores de riesgo de la obesidad infantil y se ha demostrado que la promoción y publicidad influyen en las preferencias, solicitudes de compra y consumo de los niños y adolescentes.

Respecto de los medios de comunicación de masas utilizados, se identifica la televisión (nacional y por cable) como el medio más común para transmitir promoción y publicidad dirigida a niños. Sitios web, redes sociales y juegos, la vía pública (propaganda publicitaria), puntos de venta, empaques y revistas infantiles también fueron considerados medios representativos. En Costa Rica, los puntos de venta y las redes sociales fueron mencionados como los medios más efectivos; se aduce que los niños tienen la libertad de decidir cómo invertir su dinero en los puntos de venta, por lo que la promoción y publicidad en ese momento puede ser un factor determinante en la decisión de compra. En este mismo orden de ideas, se considera que las redes sociales representan un medio novedoso para los niños y de bajo costo para las empresas.

Por su parte, las estrategias y técnicas de promoción y publicidad más atractivas para los niños fueron: uso de personajes, entrega de obsequios y uso de colores llamativos. 
En el caso de Costa Rica, se cuenta con regulaciones gubernamentales que prohíben explícitamente la venta de productos que no cumplen con los criterios nutrimentales que se establecen: “(...) no se use, coloque o exhiba propaganda comercial que promocione, directa o indirectamente, o contenga mensajes subliminales, respecto al uso, tenencia o consumo de los alimentos y productos prohibidos" (UNICEF, 2015, p. 24).

Sin embargo, algunas escuelas evaluadas no cumplen con estas regulaciones, pues se exhiben afiches de marcas comerciales, se cuenta con máquinas expendedoras de alimentos no recomendados, productos empacados en las sodas, etc. Unido a esta situación, se ofrecen actividades escolares patrocinadas por empresas de alimentos en las que se regalan entradas para el cine y cupones de alimentos, se ofrecen charlas educativas dirigidas a padres e impartidas por nutricionistas que laboran en empresas de golosinas dulces o saladas.

Respecto de la regulación gubernamental que limite o prohíba la promoción y publicidad de alimentos y bebidas dirigidas a niños en Internet, es inexistente en los tres países piloteados, entre ellos Costa Rica. La urgencia de que se regule esta red informática obedece a que el $98 \%$ de los sitios revisados incluían técnicas de promoción y publicidad dirigida a niños, el $100 \%$ estrategias de marca, el $32 \%$ ofrecía algún obsequio y el $46 \%$ de los sitios tenía juegos virtuales con los que los niños podían interactuar.

En fin, la influencia de las prácticas de promoción y publicidad de los alimentos y bebidas que se ofrece al público infantil está provocando cambios en los patrones de alimentación familiares, alterando la salud del niño y variando su percepción del sabor y sus preferencias de consumo.

\subsubsection{El vocabulario gastronómico infantil en inventarios léxicos costarricenses}

En Costa Rica, se han realizado dos estudios específicos sobre el léxico que utilizan los niños preescolares en distintas situaciones comunicativas. El primero, Léxico básico preescolar costarricense (Murillo Rojas y Sánchez Corrales, 2002), determina estadísticamente el repertorio léxico utilizado por los niños de 5 y 6 años, teniendo en cuenta una variedad de situaciones comunicativas. Se identifica el índice de uso de las palabras y su correspondiente dispersión en diez campos temáticos identificados, a saber: casa-familia, 
fantasía, juegos y juguetes, animales, alimentos, kínder, paseos, comunidad, televisión y amigos. Del total de palabras morfológicas emitidas, el 7,9 \% correspondió al campo referencial alimentos. Al revisar el inventario léxico que corresponde a la segunda parte de este estudio, se logró identificar nombres de alimentos de las siguientes categorías: golosinas, frutas, verduras, legumbres, comidas preparadas, postres y condimentos.

La valoración cualitativa de este estudio, en opinión de los autores, refleja la estrecha relación lengua-cultura-identidad-historia externa de Costa Rica. El elemento léxico más representativo lo constituye un núcleo común compartido con otras variedades de español, pues se trata de un repertorio de palabras básicas de que dispone el niño.

El segundo trabajo, Disponibilidad léxica de los niños preescolares costarricenses (Sánchez Corrales y Murillo Rojas, 2006), resalta el hecho de que los estudios sobre el léxico son de gran importancia para la planificación de la enseñanza de la lengua, pues proporcionan el vocabulario de uso de una comunidad de habla en situaciones concretas de comunicación. Este estudio indagó sobre los siguientes campos temáticos: partes del cuerpo, vestido, juegos y juguetes, alimentos, familia, animales, profesiones y oficios, comunidad, medios de comunicación y medios de transporte. Respecto del vocabulario relativo a alimentos, se señala que se recopilaron 277 palabras, en las que los términos imprecisos estaban casi ausentes. Además, se acota que "es de notar la presencia de nombres específicos que designan referentes relacionados con el gusto goloso de los niños, fomentado por la publicidad: picaritas, coca y fanta en la categoría de léxico compartido [entre niños y niñas]; merendinas, picaronas y quechitos, emitidos por los niños" (p. 52). Los veinte vocablos de mayor disponibilidad (arroz, frijoles, manzana, naranja, piña, banano, carne, papaya, sandía, uva, zanahoria, tomate, huevo, papa, pollo, sopa, pan, limón, mango, macarrones) muestran las preferencias alimenticias y gustos de los niños y, en términos generales, la dieta regular del costarricense. De las veinte palabras de mayor disponibilidad, nueve son frutas, siete tropicales y dos de reciente incorporación a la dieta del costarricense: manzana y uva; y las tres palabras que encabezan la lista son arroz, frijoles y manzana.

En síntesis, la valoración cualitativa del vocabulario gastronómico infantil necesariamente conduce a un acercamiento teórico-práctico desde la evolución del desarrollo del sentido del gusto y a la fuerte aceptación infantil de los sabores dulces, el entorno familiar 
del niño y sus hábitos de consumo, la presentación de los alimentos en espacios positivos, la función de las estrategias de promoción y publicidad de alimentos y bebidas dirigidas al público infantil, las políticas nacionales, la educación nutricional y la consideración en el ambiente educativo de la temática alimentos como objeto de estudio. En consecuencia, la apropiación del vocabulario gastronómico en el niño y su correspondiente verbalización es una tarea asumida por diferentes referentes socio-culturales con objetivos distintos.

\section{Metodología}

La presente investigación descriptivo-transversal asume el hecho de que la socialización y la adquisición del lenguaje son parte del mismo proceso; por tanto, se hace necesario observar y registrar la producción lingüística en diferentes prácticas sociales en que interactúa regularmente el niño.

Con el propósito de dar respuesta al cuestionamiento principal, ¿cuál es el vocabulario gastronómico que usan los niños en su cotidianeidad?, se siguieron tres procedimientos básicos, a saber: consulta a un corpus lingüístico, entrevistas y observaciones directas.

Del corpus oral de niños de 2 a 6 años, proyecto de investigación 745-B6-320, Test morfosintáctico para niños costarricenses menores de 6 años, se tomaron los nombres relativos a alimentos y sus respectivos contextos de uso. Este corpus está formado por textos orales producidos por niños de 2 a 6 años de edad, divididos en 8 rangos etarios, cada uno con 6 meses de diferencia, para un total de 78 niños que asistieron a la educación preescolar costarricense, durante los años 2014, 2015 y 2016.

Para dar respuesta a la pregunta, ¿cuáles son los productos alimenticios que consumen los niños con mayor frecuencia?, se realizó una entrevista a los dependientes o pulperos de 23 establecimientos donde se vende al público artículos de comercio al detalle, denominados pulperías, minisúper, supermercados, abastecedores, supermercados, ubicados en los siguientes cantones: Moravia, Goicoechea, Montes de Oca y San José. En total, se entrevistaron a 24 personas, un 56,5\% de mujeres y un 43,4 \% de hombres. La entrevista consistió en responder dos preguntas de forma oral: ¿cuáles son los productos que los niños piden que les compren? y ¿cuáles son los alimentos u otro producto comestible que los padres compran a sus hijos? 
Se aplicaron dos entrevistas más, una a niños y otra a docentes, las cuales fueron realizadas por estudiantes de la carrera de Educación Preescolar de la Universidad de Costa Rica, durante el I ciclo de 2017. La primera se realizó a 19 niños (diez niñas y nueve niños), que asistían a diferentes instituciones preescolares del país. En este caso, se hicieron tres preguntas, a saber: ¿cuál es su comida preferida? o ¿qué es lo que más le gusta comer?, ¿si tuviera que elegir algo para comer ahora, qué elegiría? y ¿qué es lo que menos le gusta comer? Las edades de los niños oscilaban entre los 2 y los 6 años.

La última entrevista se aplicó a los docentes de los niños entrevistados, quienes día a día observan los productos alimenticios que consumen sus alumnos en las meriendas. La entrevista consistió en responder cinco preguntas: ¿cuáles son los alimentos más frecuentes que consumen los niños durante la merienda (sólidos y líquidos)?, ¿qué tipo de bebidas preparadas en la casa consumen los niños?, ¿qué tipo de bebidas empacadas por la industria alimenticia consumen los niños?, ¿qué tipo de comidas preparadas en la casa consumen los niños? y ¿qué tipo de comidas empacadas por la industria alimenticia consumen los niños?

Finalmente, se realizó un registro de observación sobre los alimentos que consumieron los niños el día de la entrevista, según la siguiente categoría: alimentos sólidos y alimentos líquidos. También se registró la información relativa a la temática sobre la cual conversaban los preescolares durante el período de alimentación, a fin de saber si hacían alusión a los alimentos que estaban consumiendo en ese momento.

\section{Análisis de datos}

En este apartado, se presenta el análisis de los datos obtenidos mediante cada una de las estrategias utilizadas: revisión del corpus oral de niños de 2 a 6 años, entrevistas y observación directa, a fin de responder las siguientes preguntas:

1. ¿Cuál es el vocabulario relativo a nombres de alimentos presentes en el corpus de textos orales de niños de 2 a 6 años, proyecto de investigación 745-B6-320?

2. ¿Cuáles son los productos alimenticios que consumen los niños con mayor frecuencia?

3. ¿Cuáles son los alimentos preferidos por los niños, según su propia opinión?

4. ¿Cuáles son los alimentos que consumen los niños en las meriendas escolares? 


\subsection{Vocabulario relativo a los nombres de alimentos presentes en el corpus de textos orales de niños de 2 a 6 años, proyecto de investigación 745-B6-320, Test morfosintáctico para niños costarricenses menores de 6 años}

El vocabulario correspondiente a los nombres de alimentos se analiza siguiendo la organización interna del corpus, o sea, la producción por rangos de edad, en períodos de 6 meses, de 2 a 6 años.

En el campo referencial "nombres de alimentos y vocablos asociados", el vocabulario identificado se asocia, sin duda, a aquellos alimentos que consumen los niños durante los diferentes tiempos de comida, a los evocados a partir del estímulo del entrevistador y a sus gustos particulares. La alimentación es parte de la experiencia de vida, por tanto, el aprendizaje de este tipo de vocabulario está relacionado con la creación de oportunidades para que el niño entre en contacto con la diversidad de formas, colores, texturas, sabores y tipos de preparación de los distintos alimentos. Seguidamente, se enumeran los vocablos identificados por grupo.

-Grupo N. ${ }^{\circ}$ 1: jugo, fresa, manzana, leche, queso, huevo, pollo, fideos, pan, arroz, chocolate, confites, galletas, helados, sopa, ensalada, comida. De este grupo, cabe destacar la presencia de vocabulario relacionado con las golosinas, definida esta palabra por el DEL como un "manjar delicado, generalmente dulce, que sirve más para el gusto que para el sustento", pero apetecido por el público infantil, tal como muestran los datos de esta investigación. Se presentan los siguientes ejemplos como ilustración:

[1] *ADU: ¿Qué son?

*AND: (..) e e(s)te e(s) un compite [: confite] [*] .

[2 ] *ADU: ¿Qué compraste en el súper con mami ?

*LUC: Galletas.

[3 ] *IGN: Me quiero comer e(s)ta manta:na [: manzana] [*] .

[4 ] *ADU: ¿Qué está comiendo ?

*LUC: $\quad \operatorname{xxx}(\mathrm{en})$ salada [?] 
-Grupo N. ${ }^{\circ}$ 2: fresa, jocote, manzana, melocotón, fruta, mora, naranja, papaya, pera, uva, maní, confites, galleta, helado, pastel, mermelada, postre, arroz, cereal, pan, frijoles, salchicha, carne, huevo, leche, mantequilla, yogurt, sándwich, postre. Además, se asocian palabras como nombres de tiempos de alimentación (desayuno y comida) y otros vocablos asociados a la ingesta de alimentos: cuchara, cuchillo, plato, tapa, taza, vaso. A continuación, algunos ejemplos:

[5 ]*CAT: ¿Dónde está la flesa [: fresa] [*]?

[6 ]*ADU: ¿Y qué más te gusta comer a vos?

*EVA: Em: los jocotes no me gusta(n).

[7 ]*ADU: (.) ¿Qué es eso?

*TAM: Una manzana y eso es un chánguish [: sándwich] [*] (.) y un f(r)esco .

[8 ]*ADU: Ah bueno pero contame ¿qué hacés con tu papá ?

*MRN: $\quad$ Mi papá se compió [: compró] [*] un lelao [: helado] [*].

[9 ]*ADU: ¿Qué puedo pedir de comer que hay?

*AND: $\quad \operatorname{Arro}(\mathrm{z})$ con $\mathrm{f}(\mathrm{r}) \mathrm{ijole}(\mathrm{s}) \mathrm{y}$ huevo .

Tal como puede observarse, las frutas ocupan un lugar privilegiado en el gusto de los niños, pues mencionaron 11 vocablos relativos a este tipo de alimento y en segundo lugar sobresalen los dulces (confites, galleta, helado, pastel y postre). Ambos grupos de alimentos son producto de la dicotomía alimenticia en la infancia: las frutas son, por excelencia, el alimento que los adultos esperan que consuman los niños, en pro de una buena salud según la dieta establecida culturalmente en el país; los dulces y golosinas responden al innegable gusto de los niños por los dulces y a la fuerte publicidad dirigida a esta población, en la cual se ofrecen dulces de tamaños, formas, texturas, colores y sabores intensos y atractivos que motivan su consumo.

-Grupo N. ${ }^{\circ}$ 3: naranja, piña, uva, sandía, fruta, queso, leche, confite, galleta, granizado, sirope, papa, zanahoria, comida, hamburguesa, salsa, huevo, miel, pan. Además de los vocablos propios de este universo léxico, se incluyen dos adicionales que se asocian a la preparación y al consumo de alimentos: cuchara y tapa. Como ilustración, dos ejemplos: 
[10]*ADU: ¿Cuáles frutas?

*MON: (.) Pina [: piña] [*].

[11]*THI: $\quad$ Yo quiero un graniza(d)o (.) y unas papas .

Grupo N. ${ }^{\circ}$ : manzana, mora, naranja, pera, uva, espinacas, papa, tomate, zanahoria, chocolate, chupa-chupa, confites, gomitas, queque, pastel, miel, nutela, sándwich, salsa, sopa, jugo, leche, refresco, fresco, carne, huevo, queso, mantequilla, arroz, frijoles, pan. Se adicionan los vocablos relacionados con la preparación y consumo de alimentos: plato, vaso, tapa, taza. Al igual que en los grupos anteriores la diversidad de vocabulario incluye frutas, verduras, golosinas, postres, bebidas, alimentos preparados, productos lácteos y utensilios de cocina. Seguidamente ejemplos 12, 13, 14 y 15.

[12]*ADU: ¿Cuáles te gusta comer?

*SEB: $\quad$ xxx pues me gusta comer ra [: la] [*] naranja y ra [: la] [*] manzana y ra $[:$ la $[*]$ pera .

[13]*ADU: ¿Y por qué?

*EMMA: Po(r):que [/] (..) este (.) \&m: porque mi mamá me dio (.) este (.)

(S)opita y papas (.) y me dio una papa y yo vomité [?] en la xxx .

[14]*SEB: Mi comida favorita es alguna.

*SEB: $\quad$ El to (.) el tomate algunas veces.

*SEB: También la laranja [: naranja] [*].

*SEB: Y ta (.) también me gustan to(d)as las cosas que existen .

[15]*ISA: Gallopinto.

*ISA: Huevo.

*ISA: Mmm (..) galletas (.) de chocolate.

*ISA: Pan (.) con nutella .

*ISA: O con mantequi(lla). 
Grupo N. ${ }^{\circ}$ 5: banano, cereza, fruta, mora, avena, huevo, leche, café, natilla, queso, pescado, plátano, tomate, vegetales, zanahoria, miel, pastel, postre, arroz con leche, chicle, chocolate, chupa-chupa, confite, gomita, helado, hielo, agua, aceite, salsa. Se mantienen las frutas, las golosinas y los lácteos como productos que aparecen reiterativamente en el discurso de los preescolares. También se adicionan vocablos relacionados con el espacio donde se consumen los alimentos en las instituciones educativos: el comedor y el nombre de utensilios de cocina como taza. Se ofrecen algunos ejemplos:

[16]*ADU: ¿Con qué?

*AAR: Avena miel y leche.

[17]*ADU: ¡Qué rico!

*ADU: ¿Y tu postre favorito?

*AAR: Arroz con leche.

[18]*ADU: ¿Y tu postre favorito?

*EMI: Ay más bien las chupachupas, los confites, los chocolates, las gomitas y los chicles.

Grupo N. ${ }^{\circ}$ 6: alimento, comida, arroz, caldo, ensalada, frijoles, garbanzos, sopa, mango, manzana, melón, naranja, papaya, piña, sandía, caramelo, golosina, cebolla, vainilla, nachos, picnic. En este caso, se adiciona el nombre de comidas preparadas como ensalada, garbanzos, frijoles, arroz, esto en virtud de la experiencia del niño como miembro activo en las rutinas de alimentación en la familia y en otros espacios donde se consume, al menos, arroz, frijoles y ensalada, tres tipos de alimentos de uso diario en los hogares costarricenses. Por otra parte, se amplía el abanico de nombres de frutas producidas en Costa Rica, de bajo costo y fácil acceso -naranja, papaya, piña, melón, sandía y manzana-. A los 5 años, los nombres de hiperónimos -alimento, comida, golosina- ofrecen información sobre un manejo de vocabulario amplio, pues puede inferirse al actualizar, por ejemplo la palabra golosina, que habrá muchas otras asociadas en el lexicón mental del niño. Adicionalmente, esta información léxica ofrece datos sobre actividades familiares relacionadas con el consumo de alimentos como "picnic”. Dos ejemplos más: 
[19]*SOF: Es que esa niña quería comer muchos muchas golosinas y no [/] no se quería lavar los dientes.

[20]*ADU: ¿Vos tienes (sic) helado favorito?

*SOF: Sí.

*SOF: El de vainilla.

*ADU: ¡Qué rico!

*SOF: Y el de piña.

Grupo N. ${ }^{\circ}$ 7: fruta, fresa, limón, mora, melocotón, uva, coco, cereal, arroz, arroz con pollo, huevo, caracolitos, espaguetis, pasta-tornillitos, pasta, salsa de tomate, peperoni, hongos, culantro, albahaca, aceitunas, maíz dulce, zanahorias, sopa de pollo, sopa de carne, carne, pollo, atún, aguacate, culantro, espinacas, papas, chile, sal, azúcar, fresco, café, té, maíz, granizado, helado. Como palabras asociadas: plato, mesa, cocina, cuchara, merienda y restaurante. La diversidad de vocabulario es una característica destacable en los segmentos de vocabulario presentados, principalmente, en este grupo en el que se nombran comidas preparadas, bebidas, condimentos, vegetales, frutas y nombres de carnes; además de palabras asociadas a las actividades culinarias y el consumo de alimentos. Algunos ejemplos:

[21]*ADU: ¿Y qué te gusta de beber?

*KIM: ¿Beber?

*KIM: Me gusta \&fles flesco [: fresco] [*] de flesa [: fresa] [*].

*KIM: De mola [: mora] [*].

*KIM: De: (...) de xxx (..) y me gusta el flesco [: fresco] [*] de limón.

[22]*ADU: ¿Con qué?

*NAH: Em: con no sé.

*NAH: Ah no con (.) la pasta to(r)nillitos con salsa rosada.

*ADU: ¡Qué rico!

[23]*ADU: Ajá: 
*NAH: Ajá, pero [/] pero mira con qué me gusta la pasta.

*ADU: ¿Con qué?

*NAH: Con [x 3] cómo se llama con (.) aceitunas con maíz dulce.

[24]*ADU: ¿Qué te gusta comer?

*KIM: Me gusta comer caracolitos atún.

*KIM: Ee: me gusta comer calne [: carne] [*].

*ADU: Ajá.

*KIM: \&e: tambié:n \&car y me gusta comer sopa.

Grupo N. ${ }^{\circ} 8$ : hamburguesa, macarrones, palomitas, pan, fideos, coca; y como palabras asociadas: almorzar, colador, pulpería, refri y tapa. Por razones propias de las temáticas que abordaron los niños de este grupo de edad, los nombres de alimentos no son cuantiosos, pero vale destacar la presencia de productos ricos en carbohidratos, los cuales según la evidencia científica son gustadas por los más pequeños (Cf. Ogden, 2005). Dos ejemplos más:

[25] *ADU: ¿Con tu hermano?

*JOS: Sí y hicimos palomitas en la noche.

[26]*ANT: Después todos hicieron se sentaron en una parte ahí y hicieron.

*ANT: Fideos fideos.

En términos generales, el vocabulario gastronómico presente en el corpus objeto de estudio se caracteriza por una gran diversidad y su conformación es, grosso modo, correspondiente con los hallazgos presentados en la investigaciones citadas en el apartado Aspectos teóricos, en el sentido de que el niño gusta de alimentos dulces, razón por la cual las golosinas y los nombres de frutas sobresalen, respecto de las verduras y las comidas preparadas. En el caso de las comidas preparadas, la presencia del arroz, los frijoles y el huevo es una constante, acompañado de las pastas, denominadas por los niños con sinónimos aproximados: fideos, macarrones, espaguetis, pastas y, otro más específico, caracolitos. Respecto de las bebidas, la leche y el jugo son las más frecuentes, pero también aparece fresco, refresco, agua, té y coca. Cabe destacar también la presencia de los productos lácteos 
entre el vocabulario gastronómico de los niños: leche, queso, yogurt, mantequilla y natilla; estos productos son considerados, generalmente, por los adultos como recomendados para el crecimiento en la etapa infantil.

\subsection{Productos alimenticios que consumen los niños, según opinión de vendedores de alimentos al detalle}

Algunos de los productos, principalmente comestibles, que se venden en los pequeños comercios ubicados en los barrios, están dirigidos a la población infantil, quienes se constituyen en clientes frecuentes de un reducido inventario de mercancías. A manera de cala, se realizó una entrevista a los dependientes de 23 establecimientos (pulperías, minisúper, supermercados y abastecedores), quienes debían responder dos preguntas: 1) ¿cuáles son los productos que los niños piden que les compren? y 2) ¿cuáles son los alimentos u otro producto comestible que los padres compran a sus hijos?, con el objetivo de saber cuáles son los productos alimenticios que consumen los niños con mayor frecuencia, por propia elección y fuera de los tiempos de comida.

La respuesta a la primera pregunta se representa de la siguiente forma:

\section{Tipo de productos solicitados por los niños}

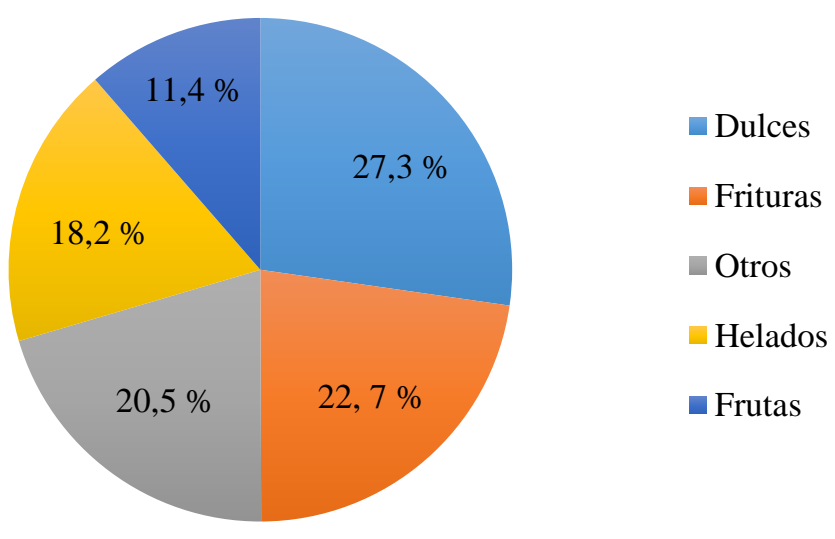

Fuente: elaboración propia, 2017. 
Entre los dulces, que corresponden al $27 \%$ del total de solicitudes de compra, sobresalen los chicles $(20,9 \%)$, pasta masticable de textura semejante a la de una goma, con sabores distintos; el limoncho (16,5\%), golosina a base de limón y sal; los popis (13,2 \%), dulce de forma redonda con un palito que le sirve de mango; el acipogo (17,6\%), un tipo de chicle ácido; la gelatina de sabores $(13,2 \%)$, golosina empacada en recipientes pequeños y con sabores dulces; los chocolates $(11 \%)$, en diferentes presentaciones, y otros $(7,7 \%)$.

Las frituras empacadas ocupan el segundo lugar de preferencia de los niños, después de los dulces. Dentro de este grupo, están las papas fritas $(20,9 \%)$, plátanos fritos $(18,7 \%)$, quesitos $(17,6 \%)$, bolitas de queso $(16,5 \%)$, tronaditas $(15,4 \%)$ y otros $(11 \%)$.

Respecto de los helados, que corresponden al 18,2 \% de las solicitudes de compra, pueden ser a base de leche, agua y en diferentes presentaciones: de paleta, en vasito y otros. Sin embargo, los más solicitados son los helados de leche, ya que fueron mencionados en un $38,5 \%$ de las veces.

Las frutas corresponden al 11,4\% de las solicitudes de compra y, básicamente, son tres: manzanas $(40 \%)$, bananos $(40 \%)$ y mandarinas $(20 \%)$.

La categoría otros, con una frecuencia de 20,5 \%, incluye los siguientes productos: palomitas de maíz (25,5\%), aperitivo elaborado a base de maíz, puede ser con mantequilla o queso, también con algún tipo de dulce; galletas (20\%), generalmente dulces y en diferentes presentaciones; pingüinos marinela $(9,1 \%)$, tipo de quequito con sabor a chocolate relleno de una crema dulce y cubierto con una pasta gruesa de chocolate; fresco leche $(18,2$ $\%$ ), bebida a base de leche con sabor a fruta, envasada en unidades de $250 \mathrm{ml}$; yogurt con chocolate, específicamente la presentación de la empresa Dos Pinos, el tipo deligurt de fresa acompañado con bolitas de arroz tostado recubiertas de chocolate y pudin con un $10 \%$ de frecuencia.

Respecto de la segunda pregunta: ¿cuáles son los alimentos u otro producto comestible que los padres compran a sus hijos?, esta decisión de los padres no es necesariamente inducida por una solicitud explícita de los niños. Las respuestas se sintetizan en el siguiente gráfico. 


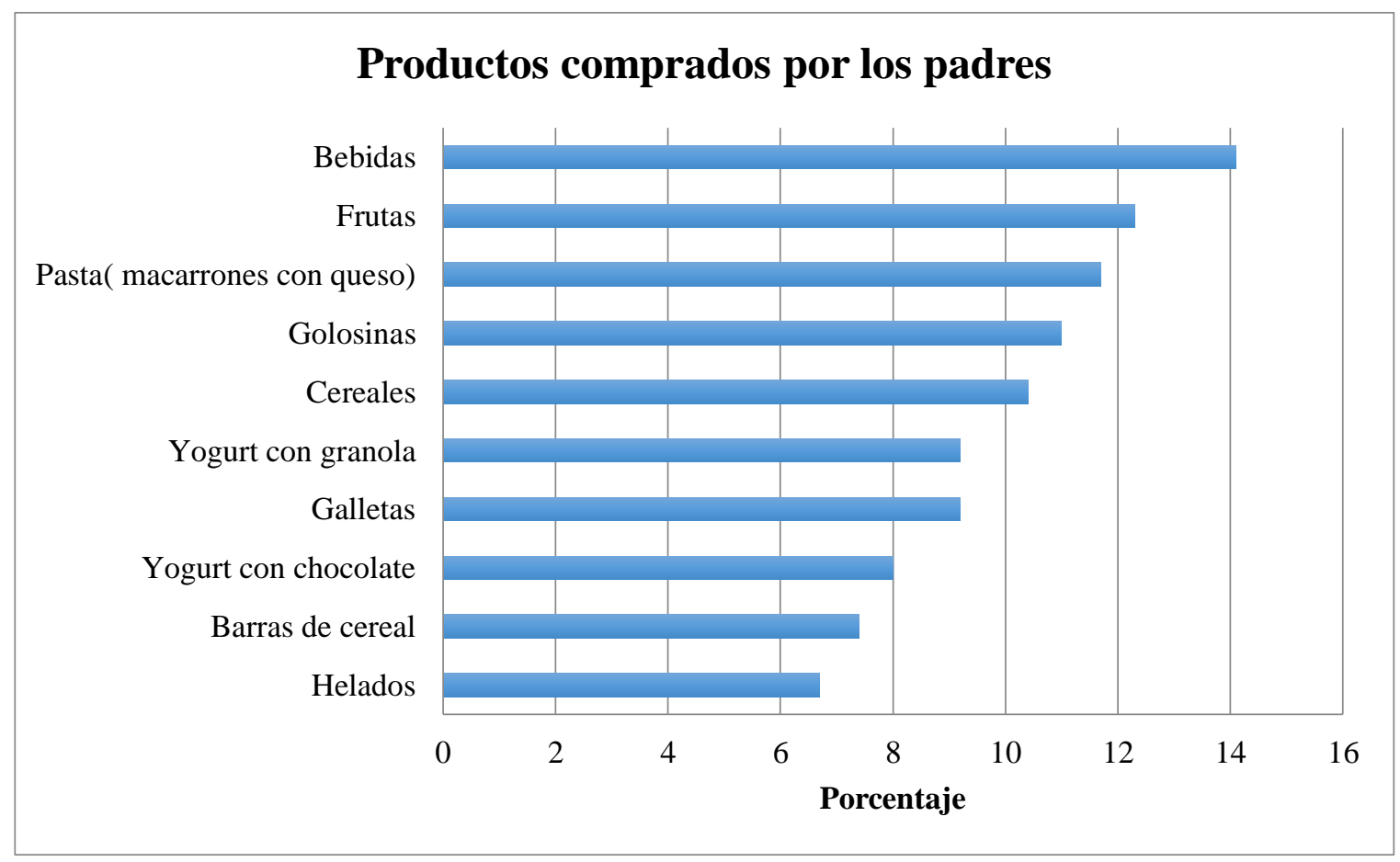

Fuente: elaboración propia, 2017.

Respecto de las bebidas, sobresalen los jugos de frutas empacados (25\%), la leche de sabores $(23,8 \%)$, normalmente envasados en unidades de $250 \mathrm{ml}$, los refrescos gaseosos $(22,5 \%)$, el yogurt $(16,3 \%)$ y la leche simple $(12,5 \%)$. Tal como puede observarse, los padres muestran preferencia por productos derivados de la leche y los jugos de fruta empacados.

Las frutas, producto alimenticio recomendado para la buena nutrición infantil, cuando es decisión de los padres adquirirlas la variedad se amplía, pues los encargados de los establecimientos comerciales mencionaron las siguientes: manzana, mandarina, fresas, bananos, uvas, mangos y kiwis; entre estas, se encuentran las tres solicitadas por los niños con mayor frecuencia: manzana, mandarina y banano. 
Respecto de los otros productos, se observa que los padres incluyen los cereales y barras de cereal, alimentos utilizados para el desayuno o para una merienda de media mañana o media tarde y las pastas semipreparadas, que en general son gustadas por los niños.

En síntesis, el vocabulario aportado por los pulperos o dependientes de tiendas de alimentos es el siguiente: chicles, limoncho, popis, acipogo, gelatina de sabores, chocolates, papas fritas, plátanos fritos, quesitos, bolitas de queso, tronaditas, helados, manzanas, bananos, mandarinas, palomitas de maíz, galletas, pingüinos marinela, fresco leche, yogurt con chocolate, pudin, jugos de frutas, leche de sabores, yogurt, leche, manzana, mandarina, fresas, bananos, uvas, bananos, mangos y kiwis, cereales y barras de cereal, pastas. Este vocabulario redunda en nombres de golosinas y frutas.

\subsubsection{Alimentos preferidos por los niños}

La elección de los alimentos por parte de los niños, jóvenes y adultos ha sido un tema recurrente de investigación, abordado desde diferentes perspectivas, a saber: los modelos evolutivos, centrados en el contacto con los alimentos, el aprendizaje social y el aprendizaje asociativo; los modelos basados en las teorías cognitivas, la motivación y los modelos de la cognición social; las perspectivas psicofisiológicas, en particular el papel de los psicofármacos y las sustancias neuroquímicas en el hambre y en saciedad, la función del estrés en la determinación de la alimentación y la alimentación excesiva (Cfr. Ogden, 2005).

La primera perspectiva, de especial interés en este trabajo, ha albergado una serie de estudios longitudinales que muestran evidencias de que los bebés recién nacidos prefieren de forma innata los sabores dulces y la sal, y rechazan los sabores amargos. Las preferencias alimenticias van cambiando en virtud de la familiaridad y la observación de otras personas que consumen alimentos. Por tanto, las actitudes de los padres frente a la comida y la elección de esta son fundamentales en el proceso de aprendizaje social. En el ámbito preescolar, los períodos de merienda también constituyen espacios de aprendizaje y socialización con los alimentos, razón por la cual se entrevistó a niños preescolares y se observó su comportamiento durante la denomina merienda, momento, a media mañana, durante el cual los niños consumen los alimentos que traen de sus hogares. 
La entrevista tuvo como objetivo indagar acerca de los gustos alimenticios de los niños, a partir del planteamiento de tres preguntas: ¿cuál es su comida preferida? o ¿qué es lo que más le gusta comer?, si tuviera que elegir algo para comer ahora ¿qué elegiría? y ¿qué es lo que menos le gusta comer?

Los dos primeros cuestionamientos están orientados hacia el reconocimiento de los gustos y el tercero se ubica en el extremo opuesto, o sea, aquello que no gusta a los niños.

Ante el primer cuestionamiento, los informantes mencionaron uno o dos nombres de alimentos, a saber: hot dog, hamburguesa, pizza, arroz, todos con frecuencia 1, arroz con pollo (frecuencia 2, en adelante únicamente se señala la cantidad de veces que aparece), pasta (1), espaguetis (1), fideos (1), macarrones (1), cereal (1), pollo (3), carne (1), huevos (1), piña (2), banano (1), frutas (2), batido de chocobanano (1), coca cola (1), yogurt líquido (1), chocolate (1) y pinto con tomate y aguacate (1). Tal y como puede observarse, a los niños les gusta comer arroz, pastas, cereales, grupo que cuenta con las frecuencias más altas, seguido de las frutas. Cabe resaltar también la presencia de las comidas rápidas, evocadas por cuatro niños.

Respecto de la segunda pregunta, cuyo objetivo fue profundizar en los gustos alimenticios de los niños y completar la información del cuestionamiento anterior, los datos muestran que se mantienen las mismas categorías de alimentos: comidas rápidas -pizza (2), papas fritas (1), palomitas (1)-, carbohidratos -arroz (1), macarrones (1) y cereales (2)-, carnes - pollo (3), carne (1)-, frutas -fresas (1), papaya (2), manzana (1) y uvas (1)-, bebidas -yogurt líquido (1) y chocolate (1)-, se adiciona la categoría vegetales mencionada por un informante y cuajada con natilla (1), tortillas de queso (1) y pinto (1).

La tercera interrogante, ¿qué es lo que menos le gusta comer?, resultó ser una fuente de gran riqueza de vocabulario, a saber: lentejas y garbanzos (1), pasta (1), arroz (1), papaya (1), melón (1), brócoli (2), arbolito (1, corresponde a la brócoli), papas (2), pepino (1), lechuga (2), pepinillo (1), cebolla (1), zanahoria (1), berenjena (1), zuquini (1), queso (1), yogurt (1) y tortillas (1). 
En consecuencia, el vocabulario gastronómico recopilado es el siguiente:

- Nombres de frutas: frutas, piña, banano, fresas, papaya, manzana, uvas, melón.

- Verduras y hortalizas: brócoli, papas, pepino, lechuga, pepinillo, cebolla, zanahoria, berenjena, zuquini, vegetales.

- Carnes: carne, pollo.

- Productos lácteos: queso, yogurt, natilla.

- Bebidas: chocolate, yogurt líquido, batido de chocobanano, coca-cola.

- Comidas preparadas: macarrones, fideos, espagueti, pasta, arroz, lentejas, garbanzos, pizza, hot dog, hamburguesa, pizza, papas fritas, tortilla de queso, tortillas, cuajada con natilla, huevo y cereal.

- Golosinas: palomitas.

\subsection{Alimentos que consumen los niños durante las meriendas escolares}

La información relativa a los alimentos consumidos por los niños durante las meriendas se recopiló mediante dos escenarios: datos aportados por docentes y la observación directa de un período de merienda en 18 instituciones educativas.

\subsubsection{Entrevista a docentes de Educación Preescolar}

La rutina de trabajo en los Jardines de Niños y en los Centros de Cuido o guarderías incluye un período de alimentación denominado "período de merienda", en el cual los niños consumen alimentos que regularmente traen de su casa. La educación preescolar considera este período como parte de la rutina pedagógica y teóricamente aprovecha este espacio para reforzar hábitos de higiene, alimentación y buenas prácticas durante la alimentación. Dadas estas circunstancias, se aplicó una entrevista a docentes relativa a la observación diaria de los productos consumidos en la merienda. $\mathrm{Al}$ respecto, se plantearon cinco preguntas:

a) ¿Cuáles son los alimentos más frecuentes que consumen los niños durante la merienda? 
Normalmente, las meriendas están constituidas por una fruta, un refresco y un tipo de pan o algún otro carbohidrato. Por tanto, ante esta pregunta, las 18 docentes mencionaron una gran diversidad de alimentos, unos más frecuentes que otros; no obstante, los más mencionados fueron: galletas (dato proporcionado por 15 docentes, en adelante únicamente se indica la frecuencia), frutas (14) y sándwich (8); seguidamente, se detallan los otros alimentos mencionados:

- Frutas: frutas (14), manzana (5), banano (4), sandía (3), mango (2), uvas (2), fresas (2), kiwi (1), papaya (1) y melón (1).

- Bocadillos: hamburguesas (1), hashbrowns (1, tipo de torta de papa frita), miniburritos (1), quesadillas (1), sándwich (8), empanadas (4), tortilla con queso (3), pancakes (2), prensadas (1), galletas con boquitas de jamón.

- Tipos de panes y cereales: galletas (15), barritas (1, entendido como barritas de cereal), quequitos (1), cereal (5), pan (1), tostadas (1), tamal de elote (1).

- Frituras: platanitos (1), tortillitas (1), pringles (tipo de papas fritas, 1), papas fritas (1).

- Dulces: pasas (1).

En vista de las jornadas ampliadas de algunos centros de educación preescolar, algunos niños deben llevar a las instituciones su almuerzo; por tanto, se mencionaron nombres de comidas preparadas, a saber: frijoles (3), arroz (3), garbanzos (1), macarrones (1), lasaña (1), verduras (2), pinto (1), picadillos (1), puré (4), huevo (3), carnes (2), pollo (1), salchichas (1), pescado (1), pollo empanizado (1).

Respecto de las bebidas, los docentes mencionaron, con las frecuencias más altas, agua (12) y jugos de caja (11), yogurt (10), frescos preparados en casa (6) y leche (6). En orden descendente y de forma más específica, se añaden: jugo de naranja (3), té frío (3), chocolate (2), licuado (1), fresco de cas (1), gelatina (1), gerber (1), tang (1), jugos de paquete (1) y sirope (1). Al reagrupar la información obtenida, se encuentra que los niños consumen mayormente bebidas preparadas por la industria alimenticia, pues jugos de caja, jugos de paquete, té frío, jugo de naranja son bebidas con una presentación similar y orientadas hacia el público infantil. Los lácteos también sobresalen, principalmente el yogurt, y, finalmente, el agua la bebida más nombrada. 
Al plantearse las otras preguntas más específicas, cuyo objetivo fue obtener información adicional que ampliara el tema de la entrevista, o sea, conocer qué consumen los niños en la escuela, pero en este caso separando los alimentos preparados en casa y los empacados por la industria. De esta manera, se obtuvo vocabulario más puntual, a saber:

- Bebidas preparadas en casa: jugo de frutas (9), fresco de naranja (2), agua (1), limonada (1), fresco de cas (1), agua dulce (1), avena (1), café (1) y jugos o refresco en polvo como tang (1), té frío (6) y otros denominados de paquete (2).

- Bebidas empacadas por la industria alimenticia: jugos de caja (29, se detallan las marcas: Del Monte, Del Valle, hi-C de la marca comercial Dos Pinos, Kerns, Caprisum, Welch y Tetra Pak), lácteos (11, leche de sabores, yogurt y leche con avena), refrescos embotellados (4, gaseosas y tropical).

Respecto de las comidas preparadas en casa, los docentes respondieron: sándwich (11), emparedados (4), gallopinto (9), arroz (5), frijoles (3), pastas (3), huevo (5), pollo (2), puré (3), quesadillas (1), papas fritas (1), burritos (1), pizza (1), lasaña (1), picadillos (1), prensadas (1), sopa (1), ensaladas (1), empanadas (1), panqueques (1), cupcakes (1), palitos de zanahoria (1), plátano (1), fruta picada (7), gelatina (3), taquitos (1), granola (1) y cereales (1).

Al focalizar la atención en las comidas empacadas por la industria alimenticia que consumen los niños, los docentes señalaron algunos tipos de comida preparadas en restaurantes de comida rápida y una serie de tentempiés o alimentos pequeños, también denominados en el medio con el anglicismo snacks. He aquí el vocabulario recopilado: galletas (15), cereales (4), barras de cereal (3), palitos de ajonjolí (2), yogurt (2), Nutri snacks (2), queques (2), sopa instantánea (2); y con frecuencia 1: comida china, pollo frito, comida chatarra y pizza, fruta empacada, licuados, gelatina, bolitas de queso, gomitas, ranchitas, barquillos, bizcochos, pingüinitos, papas Lays, quequitos, palomitas y las marcas comerciales Jacks, Pozuelo y Cosecha Dorada.

Al considerar globalmente las respuestas de los docentes y la frecuencia con que mencionaron algunos alimentos, podría afirmarse, en términos generales, que los preescolares consumen en sus meriendas mayoritariamente algún tipo de galleta, un refresco o jugo producido por la industria alimenticia y una fruta. Respecto de los almuerzos se 
observa una dicotomía entre las comidas rápidas y los alimentos propios de la dieta costarricense (arroz, frijoles y huevo).

\subsubsection{Alimentos consumidos por los niños durante las meriendas escolares}

La observación directa de un período de merienda en 18 instituciones educativas permitió registrar la siguiente información: con frecuencia 1 están las palomitas de maíz, pollo frito, burritos, granola, jamón, garbanzos, verduras, zuquini, papas, zanahoria, bizcochos, tortillas de queso, prensadas, picadillo, pan, pudines, queque de zanahoria, ranchitas, papas Lays, gomitas, maní, barritas de fruta, Nutri snack, galletas en paquete, naranja y frutas en almíbar. Las frutas consumidas en esa ocasión fueron: manzana (9), bananos (6), sandía (6), mango (5), uvas (5), papaya (3), melón (2), piña (2) y fresas (2). Además, los niños comieron galletas (16), sándwich (13), gallopinto (5), cereales (7), arroz (5), frijoles (4), macarrones (3), pescado (4), pollo (3), carnes (3), puré (3, por excelencia, puré de papa), huevo (3), tomate (3), lechuga (2), gelatina (4), palitos de ajonjolí (3), donas (2), chocolate (2), queque seco (2), empanadas (2) y merendinas y gatitos (3).

Respecto de las bebidas se consumió jugos de caja (16), productos lácteos (22, yogurt, leche, fresco leche y leche con avena), agua (11), frescos preparados en la casa (9), refrescos de botella (2), gelatina y colados, jugos en polvo y fórmula de bebé.

Finalmente, se registró el comentario o conversación que realizaron los niños durante el período de merienda, con el fin de corroborar lo señalado en otras investigaciones acerca de la importancia de las situaciones de interacción para enriquecer el vocabulario (Cf. Menti y Rosemberg, 2009).

En las observaciones realizadas los niños conversaron sobre diferentes temáticas, a saber: el recreo, la clase, los juegos, los juguetes, la familia, la ropa que vestían, pero también conversaron sobre sus alimentos. En este contexto, compararon lo que traían unos y otros, hablaron sobre lo que les gustaba o no de su merienda y compitieron para ver cuál obtenía el primer lugar, o sea, terminar de comer primero. Con esta información, podría afirmarse que si el niño lleva una merienda variada, colorida y atractiva tendrá más oportunidades para 
variar positivamente sus conductas de alimentación y de ampliar su vocabulario gastronómico.

\section{Discusión}

Para responder al cuestionamiento principal de esta investigación: ¿cuál es el vocabulario gastronómico que usan los niños en su cotidianeidad?, se integra la información recopilada mediante la consulta a un corpus lingüístico, entrevistas y observaciones directas; la triangulación de los datos dará como resultado un inventario léxico integrado que devela el vocabulario gastronómico que usan los niños en su cotidianeidad, gusten o no de los alimentos elicitados. Cuando de alimentación se trata, los niños utilizan vocabulario relativo a nombres de golosinas, frutas, comidas preparadas, bebidas, verduras, carnes, productos lácteos, cereales, nombres de marcas comerciales, condimentos e ingredientes necesarios para preparar alimentos.

La espontaneidad con la que el niño se expresa sobre temas varios, tal como muestra el corpus consultado, permitió que los informantes produjeran una variedad de vocabulario relativo a nombres de alimentos, sin que necesariamente se les solicitara que emitieran un listado de palabras sobre este campo referencial, hecho que le confiere validez al corpus consultado, del cual se obtuvo vocabulario correspondiente a ocho categorías de alimentos, que se enriquecen con la información obtenida mediante las observaciones y las entrevistas, según se presenta en el siguiente cuadro.

\section{Cuadro N. ${ }^{0} 1$}

\section{Inventario léxico recopilado}

\begin{tabular}{|c|c|c|c|}
\hline $\begin{array}{c}\text { DULCES: } \\
\text { Golosinas, frituras } \\
\text { y postres }\end{array}$ & FRUTAS & $\begin{array}{c}\text { COMIDAS } \\
\text { PREPARADAS }\end{array}$ & BEBIDAS \\
\hline $\begin{array}{l}\text { Golosinas: acipogo, } \\
\text { chicles, limoncho, } \\
\text { popis, acipago, } \\
\text { gelatina de sabores, } \\
\text { chocolates, } \\
\text { chupachupa, } \\
\text { gomitas, helados, } \\
\text { palomitas de maíz - }\end{array}$ & $\begin{array}{l}\text { Frutas: manzanas, } \\
\text { bananos, } \\
\text { mandarinas, fresas, } \\
\text { jocote, melocotón, } \\
\text { mora, naranja, pera, } \\
\text { maní, sandía, cereza, } \\
\text { limón, coco, uvas, }\end{array}$ & $\begin{array}{l}\text { Comidas variadas: } \\
\text { pasta, fideos, } \\
\text { caracolitos, } \\
\text { macarrones, } \\
\text { espaguetis, } \\
\text { tornillitos, arroz, } \\
\text { arroz con pollo, } \\
\text { frijoles, lentejas, }\end{array}$ & $\begin{array}{l}\text { Bebidas: fresco, } \\
\text { refresco, agua, té, té } \\
\text { frío, café, leche, } \\
\text { chocolate, jugo, } \\
\text { jugos de frutas, jugo } \\
\text { de naranja, leche de } \\
\text { sabores, leche con } \\
\text { avena, yogurt }\end{array}$ \\
\hline
\end{tabular}




\begin{abstract}
palomitas-, galletas, pingüinos marinela, paleta, granizado, caramelos.

Frituras: papas fritas, plátanos fritos, quesitos, bolitas de queso, tronaditas, tortillitas.

Postres: pastel, postre,

queque, pudin, gelatina, tamal de elote, cupcakes, arroz con leche.
\end{abstract}

Nota: Se menciona como nombre de algunas productos su marca, como en el caso de pringles, tipo de papa.

\section{Hiperónimo:}

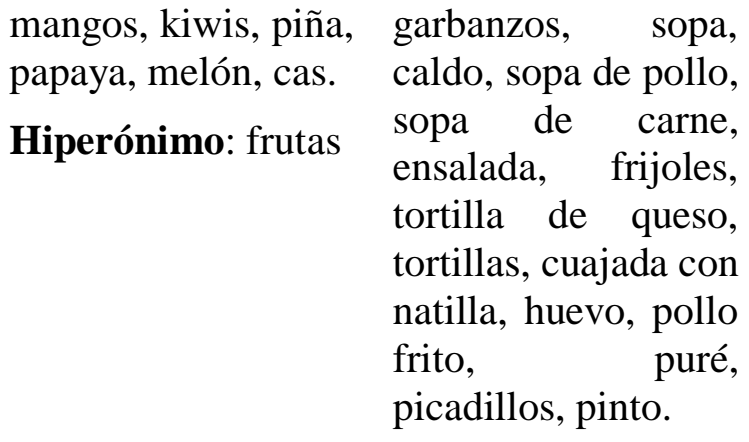

\section{Comidas rápidas:} hot dog, pizza, hashbrowns, burritos, quesadillas, prensadas, hamburguesa, sándwich, nachos, pizza, papas fritas, empanadas.

\section{Hiperónimo:}

comida, alimentos.

\section{Sinónimos} aproximados: pasta, fideos, macarrones, espaguetis.
Nota: se mencionan frescos de diferentes frutas incluidas en la columna correspondiente a frutas. golosinas, confites

\begin{tabular}{|c|c|c|c|}
\hline CARNES & $\begin{array}{l}\text { PRODUCTOS } \\
\text { LÁCTEOS }\end{array}$ & CEREALES & $\begin{array}{l}\text { VERDURAS Y } \\
\text { HORTALIZAS }\end{array}$ \\
\hline $\begin{array}{l}\text { Carnes blancas y } \\
\text { rojas: carne, pollo, } \\
\text { pescado, atún. }\end{array}$ & $\begin{array}{l}\text { Lácteos: yogurt, } \\
\text { queso, natilla, leche, } \\
\text { mantequilla, } \\
\text { cuajada. }\end{array}$ & $\begin{array}{l}\text { Panes y cereales: } \\
\text { pan, cereal, barras de } \\
\text { cereal, avena, pastel, } \\
\text { granola, tortilla, } \\
\text { tortilla de queso, } \\
\text { pancakes, tostadas, } \\
\text { palitos de ajonjolí, } \\
\text { bizcochos. }\end{array}$ & $\begin{array}{l}\text { Verduras } \\
\text { legumbres: lechuga, } \\
\text { culantro, albahaca, } \\
\text { espinacas, pepino, } \\
\text { pepinillo, cebolla, } \\
\text { zanahoria, tomate, } \\
\text { hongos, chile, } \\
\text { brócoli, zuquini, } \\
\text { papas, berenjena, } \\
\text { plátano, aguacate, }\end{array}$ \\
\hline
\end{tabular}


aceitunas,

maíz

dulce.

Hiperónimo:

vegetales

Tiempos de comida: desayuno, almuerzo, comida, merienda.

Otros: salsa, salsa de tomate, miel, sirope, mermelada, nutela, aceite, vainilla, sal, azúcar, peperoni, aceitunas.

Si bien el vocabulario perteneciente a cada una de estas ocho subdivisiones del inventario no es igualmente numeroso, sí permite visualizar la variedad de vocablos relativos a las golosinas y el estatus que ocupan las verduras en las preferencias de los niños (casi todas fueron mencionadas por ser un alimento no gustado), pero que, para efectos de vocabulario utilizado, sí debe formar parte de este inventario.

Con estos datos se corroboran los resultados obtenidos en el estudio UNICEF (2013), en el sentido de que la publicidad influye en las solicitudes de compra de los niños, tal como lo manifestaron los pulperos o dependientes, pues los pequeños compradores solicitan básicamente dulces, helados y frituras, tendencia hacia el consumo de productos calóricos que se ve reforzada por las familias, las cuales optan por meriendas en las que están presentes las galletas, los jugos o refrescos industriales y, en algunos casos, una fruta.

Otro de los aspectos por resaltar, en lo correspondiente a los nombres de comidas preparadas, es el innegable gusto de los niños por las pastas, pues las mencionan utilizando todos los sinónimos aproximados de que disponen: fideos, macarrones, espaguetis, pasta, caracolitos y tornillitos, todos con formas distintas, pero con una base común: pasta de harina.

Respecto de los alimentos preparados llama la atención la variedad de comidas rápidas que se han incorporado a la dieta de la niñez costarricense y como consecuencia en su vocabulario. Por otra parte, es positivo, desde el punto de vista de la relación lenguacultura-identidad, que los ingredientes básicos de un casado (plato combinado costarricense) forman parte del vocabulario gastronómico de los niños: arroz, frijoles, huevo, plátano, tortillas, algún tipo de carne y alguna verdura, comúnmente en picadillo. 
Al cotejar los datos presentados por Sánchez y Murillo (2006), en torno del campo semántico alimentos, del cual se obtienen un total de 277 vocablos emitidos por niños preescolares, ciclo de transición, con una metodología de trabajo distinta, las veinte palabras con mayor índice de disponibilidad (arroz, frijoles, manzana, naranja, piña, banano, carne, papaya, sandía, uva, zanahoria, tomate, huevo, papa, pollo, sopa, pan, limón, mango y macarrones) están presentes en el inventario presentado en la Cuadro N. ${ }^{\circ} 1$.

La confluencia de estos datos permite ofrecer recomendaciones a los docentes de educación preescolar, sustentadas en las prácticas de alimentación cotidiana de los niños y en la identificación del vocabulario que utilizan al expresarse sobre esta temática, en el sentido de que la mediación pedagógica debe considerar que el niño utiliza vocabulario diverso y que, indiscutiblemente, muestra preferencia por ciertos alimentos, razón por la cual es conveniente permitirles descubrir otros.

Desde una perspectiva educativa, los tiempos de comida son espacios propicios para el enriquecimiento del vocabulario y la creación de oportunidades para que el niño perciba, saboree, toque y huela los alimentos que forman parte de las rutinas obligatorias en los procesos formativos durante la infancia.

\section{Conclusiones}

Los datos obtenidos mediante esta investigación no solo reflejan los gustos gastronómicos de los niños sino también la influencia de los espacios de socialización familia e institución educativa- y la importancia que la industria alimenticia otorga a este sector de la población. En consecuencia, el aprovechamiento del inventario léxico recopilado, sin duda alguna, será versátil y dependerá de los intereses del usuario. Por ejemplo, para la educación preescolar es útil conocer cuál es el vocabulario gastronómico que conoce el niño, para enriquecerlo y resignificar los espacios de alimentación, de tal forma que el niño sea consciente de la percepción del sabor, la textura y la calidad de los alimentos, con el propósito de que, a corto plazo, logre definir con propiedad sus preferencias de consumo. En este mismo orden, los productores de alimentos industriales tendrán respuestas sobre sabores, texturas, formas de presentación, etc., que les permitirá tomar decisiones más apegadas a las preferencias gastronómicas de los niños. Los nutricionistas, por su parte, cuentan con 
información detallada para realizar ejercicios de educación nutricional basada en las preferencias de los niños costarricenses.

La lingüística aplicada, educativa y clínica cuentan con un vocabulario idóneo para elaborar evaluaciones del lenguaje infantil en las que la comprensión oral no sea un factor que interfiera, ya que se trata de un vocabulario que el niño oye a diario, que comparte con los padres y los compañeros de juego, que recibe mediante los medios de comunicación y que es capaz de emitir juicios de valor en torno de este.

\section{Bibliografía}

Aguado, Gerardo. (2000). El desarrollo del lenguaje de 0 a 3 años. Bases para un diseño curricular en la Educación Infantil (2. ${ }^{\mathrm{a}}$ ed.). Madrid: CEPE, S.L.

Bruner, Jerome. (1983). El habla del niño. Buenos Aires: Paidós.

Birch, L. L. y Fisher, J. O. (1998). Development of eating behaviors among children and adolescents. Pediatrics, 101 (Supplement 2), 539-549.

Instituto de Nutrición de Centro América y Panamá (Incap). (s. f.). Alimentación del niño y la niña preescolar y escolar. Recuperado de www.incap.int/index.php/es/.../603-3alimentacion-del-nino-preescolar-y-escolar-pdf

Moreno Villaresa, José Manuel y Galiano Segovia, M. J. (2006). El desarrollo de los hábitos alimentarios en el lactante y el niño pequeño. Sentido y sensibilidad. Revista Pediatría de Atención Primaria, VIII (Suplemento I), 11-25. Recuperado de http://www.pap.es/FrontOffice/PAP/front/Articulos/Articulo/_IXus51_LjPpy3lkHe V2u3MIcb7DuVCQg

Murillo Rojas, Marielos y Sánchez Corrales, Víctor. (2002). Léxico básico de los niños preescolares costarricenses. San José, Costa Rica: Editorial de la Universidad de Costa Rica.

Ogden, Jane. (2005). Psicología de la alimentación. Comportamientos saludables y trastornos alimenticios. Madrid, España: Morata. 
Rubio Navarro, Lorena; Torrero, Carmen; Regalado, Mirelta y Salas, Manuel. (2013). Desarrollo de la discriminación a los sabores. Neurobiología, Revista electrónica, 4 (7), $1-13$. Recuperado de https://www.uv.mx/eneurobiologia/vols/2013/7/LRubio/RubioNavarro4(7)280613.pdf

Sánchez, Víctor y Murillo, Marielos. (2006). Disponibilidad léxica de los niños preescolares costarricenses. San José, Costa Rica: Editorial de la Universidad de Costa Rica.

Smith, David y Margokskee, Roberto. (2001). El sentido del gusto. Investigación y Ciencia, 296, 4-13.

Tomasello, Michael. (2005). Constructing a Language: A Usage-Based Theory of Language Acquisition. London: Harvard University Press.

UNICEF. (2013). Promoción y publicidad de alimentos y bebidas no saludables dirigida a niños en América Latina y el Caribe. Recuperado de https://www.unicef.org/lac/Estudio_exploratorio_promocion_alimentos_no_saludab les_a_ninos_en_LAC_-_Informe_Completo_(2).pdf

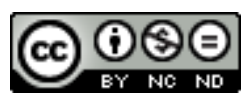

Esta obra está bajo una licencia de Creative Commons Reconocimiento-NoComercial$\underline{\text { SinObraDerivada } 4.0 \text { Internacional }}$ 